\title{
Effectiveness of D-mannose, Hibiscus sabdariffa and Lactobacillus plantarum therapy in prevention of infectious events following urodynamic study
}

\author{
Riccardo Milandri, Matteo Maltagliati, Tommaso Bocchialini, \\ Chiara Del Prete, Giampaolo Bianchi, Bernardo M Rocco \\ and Salvatore Micali
}

\begin{abstract}
Introduction and aim: The urodynamic study is an invasive examination that allows a thorough evaluation of the functional activity of the lower urinary tract (bladder, urethra). The execution of urodynamic study exposes the patient to the risk of contracting infections of the lower urinary tract. Prevention of urinary tract infections consists in the avoidance of risk factors and prophylaxis with antimicrobial and non-antimicrobial measures. In this article, we aimed to evaluate the effectiveness of a phytotherapeutic product composed of D-mannose, Hibiscus sabdariffa, and Lactobacillus plantarum in the prevention of infectious events following invasive urodynamic examination.

Materials and methods: We selected 100 female consecutive patients (age 19-87years) that underwent urodynamic invasive procedure. We prescribed I4-day therapy with D-mannose, $H$. sabdariffa, and $L$. plantarum to these 100 patients after urodynamic invasive test. After that, we have examined urine cultures and urinary symptoms through telephone interviews and hospital outpatient visits to evaluate the possible presence of urinary tract infections.

Results: Urinary tract infections were observed through urine culture in 13\% of the selected patients; of the 13 urinary tract infections, 9 were observed in patients with urinary tract recurrent infections in the last year and the other 4 cases were associated with patients with no urinary infection in the last year. Three women reported some urinary symptoms and underwent antibiotic therapy. The other 10 cases were classified as asymptomatic bacteriuria. Three cases were related to patients presenting with pelvic organ prolapse. Eight cases were instead related to significant post-voiding residue $(>100)$. Six patients with urinary tract infection reported constipation in the last year. All patients completed the prescribed therapy due to the affordable price of the product and no side effects have been reported.

Conclusion: Our study about the administration of D-mannose, $H$. sabdariffa, and $L$. plantarum after invasive urodynamic examination underlined how this phytotherapeutic product can reduce the risk of bacteriuria and urinary tract infection in women.
\end{abstract}

\section{Keywords}

Urodynamic study, urinary tract recurrent infections, D-mannose, Ellirose ${ }^{\mathrm{TM}}$, L. plantarum

Date received: 4 May 2018; accepted: 10 August 2018

\section{Introduction}

The urodynamic study (UDS) is an invasive examination that allows a thorough evaluation of the functional activity of the lower urinary tract (bladder, urethra). UDS evaluates the behavior of the urinary system during the voiding
Department of Urology, University of Modena and Reggio Emilia, Modena, Italy

Corresponding author:

Riccardo Milandri, Department of Urology, University of Modena and Reggio Emilia, Via del Pozzo 7I, 4II 24 Modena, Italy.

Email: riccardomilandri85@gmail.com 
act. More precisely, it analyzes the urinary stream during voiding and the pressure in the bladder and abdomen.

UDS is performed as a second-level diagnostic study in patients suffering from functional diseases such as urinary urgency, urinary incontinence (urge, stress, or mixed), neurological diseases, or in the outcome of bone marrow injuries. UDS consists of four distinct phases:

\section{Uroflowmetry;}

Pressure-flow study (voiding phase);

Cystometry (filling phase);

Urethral profilometry.

In specialized centers, it is also possible to undergo two other phases of the examination: the electromyography of the pelvic floor muscles and the video UDS.

To perform UDS, the patient is placed in a supine position and subjected to the introduction of a bladder catheter and a rectal probe, equipped with appropriate sensors at the extremities. With the subsequent introduction of physiological solution, sensitivity, capacity, stability, and compliance of the bladder musculature are assessed by recording intravesical pressures. It is also possible to evaluate how the patient feels the urinary stimulus, providing useful information on the functioning of the bladder.

The execution of UDS exposes the patient to the risk of contracting infections of the lower urinary tract. For this reason, the patient must necessarily bring into view a negative urine culture performed in the previous 15 days or must already be taking targeted antibiotic therapy.

Urinary tract infections (UTIs) consist in the presence of microbial pathogens in the urinary tract and represent one of the most common bacterial infections. ${ }^{1}$ Asymptomatic urinary bacteriuria $(\mathrm{ABU})$ consists in the urinary growth of bacteria in an asymptomatic individual. $\mathrm{ABU}$ requires no treatment unless the patient is subjected to urological surgery or is pregnant. ${ }^{2}$

European Association of Urology (EAU) classification of UTIs includes uncomplicated UTIs, complicated UTIs, urinary tract recurrent infections (rUTIs), catheter-associated UTIs, and urosepsis. ${ }^{2}$ rUTIs consist in recurrences of uncomplicated and/or complicated UTIs with a frequency of at least three UTIs/year or two UTIs in the last 6 months. About $20 \%-30 \%$ of women with a first UTI will have a recurrence and $5 \%$ will have chronic recurring infections. ${ }^{3}$ Clinically, UTIs can manifest as urethritis, cystitis, or pyelonephritis. Almost half of all women will experience at least one episode of cystitis during their life. ${ }^{1}$

For young women (premenopausal), the risk factors are sexual intercourses, use of spermicide, a new sexual partner, a mother with a history of UTIs during her childhood, and blood antigen secretory status. For elderly women (postmenopausal), the following can be considered as risk factors: history of UTIs before menopause, urinary incontinence, atrophic vaginitis, cystocele, increased post-void urine volume, blood group, antigen secretory status, urine catheterization, functional status, and deterioration in elderly institutionalized women. ${ }^{4}$

The spectrum of etiological agents includes Escherichia coli in $70 \%-95 \%$ of cases, Staphylococcus saprophyticus in $5 \%-10 \%$ of cases, and occasionally other enterobacteriaceae such as Proteus mirabilis and Klebsiella. Bacteria can be considered normal inhabitants of the gastrointestinal tract (the most important reservoir of uropathogenic $E$. coli) where they play an important role in the maintenance of health and development of diseases. ${ }^{5}$

Urogenital colonization by $E$. coli is feasible to a series of predisposing factors, hygiene habits, and sexual practices. In most cases, female urethra gets infected by fecal material containing uropathogenic bacteria according with the short distance between the female urethra and the anus that facilitates contamination. ${ }^{6}$

Diagnosis should be confirmed by urine culture: a colony count of $10,000 \mathrm{cfu} / \mathrm{mL}$ of uropathogens is microbiologically diagnostic in women who present lower urinary tract symptoms as dysuria, frequency, urgency, and suprapubic pain with the absence of vaginal discharges or irritation.

Prevention of rUTIs consists in the avoidance of risk factors and prophylaxis with antimicrobial and non-antimicrobial measures, unfortunately associated with the onset of resistance to the antibiotic. Non-antimicrobial prophylaxis includes the administration of probiotics, Cranberry, and D-mannose.

In particular, Lactobacillus probiotics and D-mannose are included in the phytotherapeutic drug preparation associated to Hibiscus sabdariffa. The clinical use of the phytotherapeutic drug is based on the molecular effects of its components and on their synergistic effects. D-mannose has an important role in the prevention of rUTIs. It is a sugar with a relevant role in human metabolism, especially in glycosylation of certain proteins. The supposed mechanism of action is inhibiting bacterial adherence to uroepithelial cells. ${ }^{7}$

\section{Materials and methods}

The phytotherapeutic drug is composed of D-mannose $(1000 \mathrm{mg}), H$. sabdariffa $(200 \mathrm{mg})$, and Lactobacillus plantarum Lp-115 (1 mld UFC). It can be used in non-antibiotic prophylaxis of rUTIs and its posology is two sachets daily (one in the morning and one in the evening) in the presence of urinary symptoms or after an invasive outpatient examination or one sachet daily in other clinical situations. It can be administrated for prolonged time for its generally minimal side effects.

In this study, we aimed to evaluate the effectiveness of D-mannose, H. sabdariffa, and L. plantarum in the prevention of infectious events following invasive urodynamic examination. We selected 100 female consecutive patients (age 19-87years) that underwent urodynamic invasive procedure. Patients were chosen based of the absence of 
important chronic disease, malformations of the urinary tract, and UTIs at the moment of the procedure.

Of the total, 23 had various episodes of rUTIs associated to urinary tract symptoms; 35 patients complained constipation; 32 had anatomical alteration of the pelvic organs, like colpocele, cystocele, and rectocele; 5 patients were affected by neurological diseases like multiple sclerosis (3), Parkinson's syndrome (1), and cauda equina syndrome (1). We discovered also a significant post-micturition residue $(>80 \mathrm{~mL})$ in 25 women (Table 1).

We prescribed 14-day therapy with D-mannose, H. sabdariffa, and L. plantarum to these 100 patients after urodynamic invasive test. After that, we have examined urinecoltures and urinary symptoms through telephone interview and hospital outpatient visits to evaluate the possible presence of rUTIs.

\section{Results}

UTIs were observed through urine culture in $13 \%$ of the selected patients. More specifically, 9 of the 13 UTIs were observed in patients with rUTIs in the last year and the other 4 cases were associated with patients with no urinary infection in the last year.

Only three women reported some urinary symptoms (dysuria, frequency, urgency, or pain) and they underwent antibiotic therapy. The other 10 cases were classified as asymptomatic bacteriuria (no further urinary symptoms despite the positive urine culture).

Three cases of UTIs were related to patients presenting with pelvic organ prolapse and eight cases were instead related to significant post-voiding residue $(>100)$. Six patients with UTIs reported constipation in the last year (Table 2).

All patients completed the prescribed therapy due to the affordable price of the product and no side effects have been reported.

\section{Discussion}

This phytotherapeutic drug is a dietary supplement (combination of Lactobacillus probiotics, D-mannose, and $H$. sabdariffa) widely used by the urologists in our region. The absence of side effects makes it easily and safely administrable for a long period, especially for non-antimicrobial prophylaxis in rUTIs, without risk of the development of any antibiotic resistance.

Kranjčec et al. ${ }^{8}$ investigated the role of D-mannose in the prevention of UTIs in women with history of rUTIs initially treated with antibiotic therapy. $H$. sabdariffa is a common herbal drink consumed both hot and cold by people around the world and used in traditional medicine for the treatment of hypertension and UTIs.

I Alshami et al. showed that $H$. sabdariffa extract significantly inhibits uropathogenic growth and prevents the "in vitro" biofilm-forming capacity of bacteria like E. coli
Table I. Population considered in the study.

\begin{tabular}{ll}
\hline Population of 100 women & Rate (\%) \\
\hline rUTI & 23 \\
Constipation & 35 \\
Pelvic organ prolapse & 32 \\
Neurological disease & 5 \\
Significant post-voiding residue & 25 \\
\hline
\end{tabular}

rUTI: urinary tract recurrent infection.

Table 2. Results of the study.

\begin{tabular}{lc}
\hline Infectious events and risk factors & Rate (\%) \\
\hline Positive urine culture & 13 \\
- UTI & 3 \\
- Bacteriuria & 10 \\
- rUTIs in the last year & 9 \\
- No UTIs in the last year & 4 \\
Risk factors (in positive urine culture patients) & \\
- Pelvic organ prolapse & 3 \\
- Significant post-voiding residue $(>100)$ & 8 \\
- Constipation & 6 \\
\hline
\end{tabular}

and Klebsiella pneumoniae. The antimicrobial activities of the extract observed provided basic evidence for the potential antimicrobial effects of this plant for preventing rUTIs. ${ }^{9}$

UDS is an invasive procedure that exposes patients to risk of UTI. Nóbrega et al. ${ }^{10}$ underlined that the incidence of bacteriuria, transient bacteriuria, and UTI in 232 women after UDS was $11.6 \%, 7.3 \%$, and $4.3 \%$, respectively.

D-mannose, H. sabdariffa, and L. plantarum can be used in prophylaxis to reduce the risk of UTI after invasive procedure like urodynamic studies. Nadeem et al. ${ }^{11}$ reported that $14 \%$ of the patients who underwent urodynamic test had a positive urine culture and $12 \%$ required treatment with antibiotics. In our study, we observed that the combination of D-mannose, $H$. sabdariffa, and L. plantarum reduces the risk of symptomatic UTI.

Moreover, as is already known from the literature ${ }^{10,11}$ we ascertained how some conditions like post-voiding trace and pelvic anatomical alterations can predispose to asymptomatic bacteriuria or UTIs in women. Of course, this study presents several limitations mainly because this is a single-center, single-arm, uncontrolled observational study.

\section{Conclusion}

Our study about the administration of D-mannose, $H$. sabdariffa, and $L$. plantarum after invasive urodynamic examination underlined how this phytotherapeutic product can reduce the risk of bacteriuria and UTI in women. Certainly, further randomized controlled studies are needed in order to establish the efficacy with a sufficient level of evidence. 


\section{Declaration of conflicting interests}

The author(s) declared no potential conflicts of interest with respect to the research, authorship, and/or publication of this article.

\section{Funding}

The author(s) received no financial support for the research, authorship, and/or publication of this article.

\section{References}

1. Foxman B. Epidemiology of urinary tract infections: incidence, morbidity, and economic costs. Dis Mon 2003; 49(2): 53-70.

2. Johansen TE, Botto H, Cek M, et al. Critical review of current definitions of urinary tract infections and proposal of an EAU/ESIU classification system. Int J Antimicrob Agents 2011; 38: 64-70.

3. Hooton TM. Recurrent urinary tract infection in women. Int $J$ Antimicrob Agents 2001; 17(4): 259-268.

4. Naber KG, Schito G, Botto H, et al. Surveillance study in Europe and Brazil on clinical aspects and Antimicrobial Resistance Epidemiology in Females with Cystitis (ARESC): implications for empiric therapy. Eur Urol 2008; 54: 11641175.
5. Hansson S, Jodal U, Lincoln K, et al. Untreated asymptomatic bacteriuria in girls: II - effect of phenoxymethylpenicillin and erythromycin given for intercurrent infections. BMJ 1989; 298: 856-859.

6. Yamamoto S, Tsukamoto T, Terai A, et al. Genetic evidence supporting the fecal-perineal-urethral hypothesis in cystitis caused by Escherichia coli. J Urol 1997; 157(3): 1127-1129.

7. Schaeffer AJ, Chmiel JS, Duncan JL, et al. Mannosesensitive adherence of Escherichia coli to epithelial cells from women with recurrent urinary tract infections. $J$ Urol 1984; 131: 906-910.

8. Kranjčec B, Papeš D and Altarac S. D-mannose powder for prophylaxis of recurrent urinary tract infections in women: a randomized clinical trial. World J Urol 2014; 32: 79-84.

9. Alshami I and Alharbi AE. Antimicrobial activity of Hibiscus sabdariffa extract against uropathogenic strains isolated from recurrent urinary tract infections. Asian Pac J Trop Dis 2014; 4: 317-322.

10. Nóbrega MM, Auge AP, de Toledo LG, et al. Bacteriuria and urinary tract infection after female urodynamic studies: risk factors and microbiological analysis. Am J Infect Control 2015; 43(10): 1035-1039.

11. Nadeem M, Sheikh MI, Sait MS, et al. Is urinary tract infection after urodynamic study predictable? Urol Sci 2017; 28(4): 240-242. 